\title{
ProsocialLearn: a Prosocial Games Marketplace
}

\author{
Francesco D'Andria, Jose Miguel Garrido \\ ATOS Spain SA \\ Madrid - Spain
}

\author{
Michael Boniface, Stefano Modafferi, Simon Crowle, \\ Lee Middleton \\ University of Southampton IT Innovation Centre, \\ Southampton, UK
}

\begin{abstract}
ProsocialLearn is a digital pro-social games platform. The ProsocialLearn project has delivered a series of disruptive innovations for the production and distribution of prosocial digital games that engage children (7-10 years old). Additionally it has stimulated technology transfer from the games industry to the educational sector. ProsocialLearn fosters the creation of a new market for digital games aimed at increasing social inclusion and academic performance, as well as a distribution channel to deliver prosocial games to children and teachers in European schools. Furthermore, it provides a proven pro-social methodology to design digital games. The ProsocialLearn platform makes available a series of APIs, which game developers use to integrate many of the ProsocialLearn functions into games, i.e. emotion and engagement monitoring, in-game achievements, games adaptation based on Prosocial Learning Objectives (PLOs), and micro-transactions.
\end{abstract}

Keywords- pro-social games, digital gaming platform, monitoring pro-social skills.

\section{INTRODUCTION}

Social inclusion is a key concept in European social policy, and both the Europe 2020 strategy and the Digital Agenda for Europe aim to ensure greater social cohesion and employment.

Support for disengaged and disadvantaged learners, enhancing their employability and integration into society is a key objective. This includes helping people with learning disabilities and young people to be more employable. Children in danger of social exclusion due to showing reduced empathy or high levels of aggressive or anti-social behaviours should benefit from digital games tailored to teach prosocial skills. These can help them to achieve academically, appreciate team work and recognize the value of understanding other people's needs. However, current digital games targeting the education sector are low quality and fail to capture the imagination of players, significantly reducing their effectiveness. Contrast this to games in the entertainment industry where innovative technologies and delivery models are revolutionising society and culture. It is clear that traditional game designers know how to produce engaging stories and game content but they are lacking scientifically proven game mechanics that can be used to create serious games for non-leisure contexts in a way that delivers beneficial outcomes for players.

\author{
Konstantinos C. Apostolakis, Kosmas Dimitropoulos, \\ Petros Daras \\ Information Technologies Institute - ITI \\ Centre for Research and Technology Hellas - CERTH \\ Thessaloniki, Greece
}

ProsocialLearn (http://prosociallearn.eu/) studies how to create and deliver digital games for children (7-10 years old) within educational systems that support learning of prosocial skills. The approach combines prosocial pedagogies with advanced ICT technologies and cloud delivery models to create attractive and exciting learning opportunities for children based on digital games. On the other hand, ProsocialLearn aims at establishing a new market for digital games targeting at increasing social inclusion and academic performance.

\section{A. ProsocialLearn project challenges}

In-depth the main challenges of the ProsocialLearn project are therefore:

a) The creation of a new ecosystem for student learning and skill acquisition based on Prosocial Gaming that channels creativity, innovation and technologies from the traditional gaming industry to the education sector. The traditional game industry is thriving with ideas and technical solutions that can directly compliment and benefit serious games, however, the financial risk to small game companies must be significantly reduced to incentivise new game productions by offering domain specific expertise, marketing and distribution channels for digital games. The perception that games are for entertainment must be overcome to increase acceptance of their use by teaching professionals in school curricula.

b) The provisioning of a dedicated prosocial game development and distribution platform to distribute prosocial digital games from SME game companies to the education sector. The ProsocialLearn platform aims at supporting developers by providing scientifically proven prosocial game elements that can be used to develop games targeting children for Prosocial Learning through an API that allows them to integrate functions (visual sensing, identification of prosocial signals from in-game actions, personalised adaptation of game elements, player profiles, game mechanics and expressive virtual characters) into digital games, whilst supporting mechanisms for data collection with protection of personal information.

c) A prosocial gamification methodology for learning and skills acquisition using serious games will be offered based on a rigorous multidisciplinary approach that involves serious design and implementation grounded in scientific evidence in 
order to consistently deliver efficient content able to serve the required pedagogic goals.

\section{B. ProsocialLearn project results}

ProsocialLearn's main results are the establishment of a new ecosystem for student learning and skill acquisition based on prosocial gaming that channels creativity, innovation and technologies from the traditional games industry to the educational sector.

The financial risk to small game companies must be significantly reduced to create an incentive for new prosocial game productions, by offering domain-specific expertise, marketing and distribution channels for these games, and the perception of games must also be changed to increase acceptance of their use by teaching professionals in school curricula. Production and distribution of prosocial games A dedicated prosocial game development and distribution platform must be provided to distribute prosocial digital games from SME game companies to the educational sector.

ProsocialLearn's innovative API will allow developers to integrate a range of functions into prosocial digital games, though supporting mechanisms for data collection and protection of personal information. A prosocial gamification methodology for learning and skills acquisition using serious games must also be provided in order to consistently deliver effective content that serves the required pedagogic goals.

\section{Validation of the ProsocialLearn platform}

Student outcomes must be linked to prosocial learning objectives to communicate expectations and to provide the basis for evaluating teacher, student and game effectiveness.

Prosocial skills must be measured efficiently and costeffectively using a series of informational cues related to gameplay behaviour and signals hidden in game mechanics will be linked to prosociality. Using a pedagogically sound prosocial model that stores student data over time, teachers are able to efficiently monitor progress and assess mid- to longterm learning outcomes across multiple games and game sessions.

In the context of ProsocialLearn, games are aligned to students curricula, helping teaching professionals to understand how to incorporate games into teaching programmes. A series of studies have been conducted in real schools throughout Europe using games produced by the five Gaming Providers that are part of the consortium. Those studies aimed at evaluating mid- and long-term social and academic effectiveness of prosocial gaming is provided as feedback to policy makers. All experiments are supervised by an ethical oversight procedure and ethics advisory board, active over the whole duration of the project.

\section{PLATFORM OVERVIEW}

Acquiring skills for social and emotional well-being is important for inclusive societies and academic achievement. Studies have demonstrated the beneficial link between prosocial behaviours and improved results in curriculum topics [8]. The primary purpose of the platform is to enable a prosocial learning process for creation and delivery of digital games for children (7-10 years old) within educational systems that support learning of prosocial skills. The approach combines prosocial pedagogies with advanced ICT and cloud delivery models to create attractive and exciting learning opportunities for children; produce novel digital game-based pedagogies and simplify deployment within educational organisations.

Prosociality is a concept that refers to an individual's propensity towards positive social behaviours. Individuals with prosocial skills are, for example, able to join in conversations, talk nicely, identifying feelings and emotions in themselves and others, identify someone needs help and ask for help. ProsocialLearn classifies these skills in terms of Friendship, Feelings and Cooperation. By using interactive digital games supported by additional instructive and reflective activities, ProsocialLearn allows children to learn social skills that can be generalised to real life situations in the classroom, playground and at home. ProsocialLearn adopts a skills based-approach to learning social skills. We have identified an initial set of 40 skills within three classes: skills for friendship, skills for feelings, and skills for collaboration [1]. The skills were selected considering their applicability to and benefit from digital game-based learning, for example, the skill can be measured through sensor observation and monitoring tools.

The skills are also of different difficulties and can be incrementally learnt to progress students through levels of prosociality. For example, identifying feelings is necessary to be able to showing concern for other's feelings or dealing with angry feelings. Each game can be used to learn one or more skills depending on the nature of the game situations, decisions and mechanics.

The adoption of digital games by the formal education sector requires significant innovation in practices of formal schooling, and in the procurement and certification systems for education products. Issues such as the lack of community of practice and time to prepare lessons based on games, the perception that the game takes over from the teacher and incompatibility with teaching practices and general fit to the curriculum are some of the challenges faced by games companies targeting the sector.

Our approach to resolving these problems is to provide a platform supporting the multidisciplinary, co-creation of social games in a way that bridges the gap between communities of teachers and game developers, whilst accumulating evidence for the benefits of social games within schools. Fig. 1 shows the ecosystem for the proposed platform. Teachers access games and work with game designers to create and share usage scenarios, lesson designs and success stories within educator networks, promoting adoption and best practice for the wide variety of educational settings expected in schools. The platform operator's business models are based on revenues derived from interactions between users (schools and games companies). The platform itself has little or no value for the initial users and we adopt an appropriate a seeding strategy for engaging users in what is an unknown business proposition initially without critical mass. The strategy focuses on establishing a set of exemplar games created by teachers and 
games companies that used to build evidence and are promoted through regional ambassadors. The goal is then to rapidly grow the network on a global scale and remove all barriers to interaction. How the interaction translates to revenue is dependent on the adopted business model. If teachers cannot or will not pay directly (for example pay-per-use or subscription) then other sources of funding are needed such as advertising, sponsored games, or supporting loss leaders.

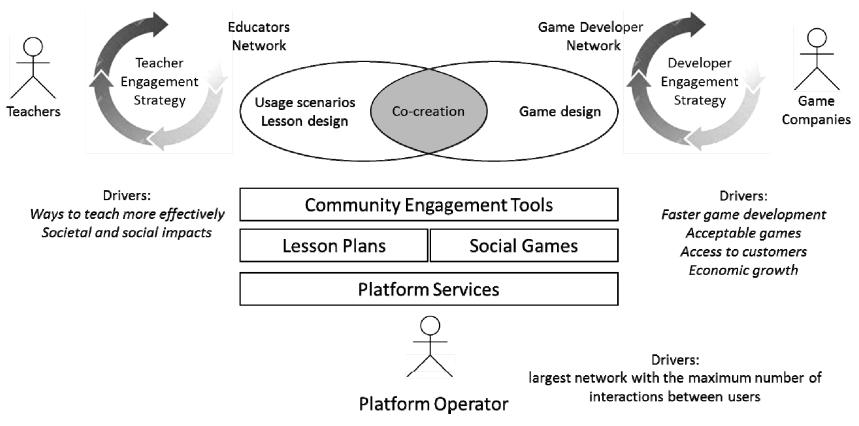

Fig. 1 High level concept of the ProsocialLearn platform

\section{Platform Functional Overview}

ProsocialLearn platform is implemented through a technology platform offering systematic pedagogical support for prosocial games developed by an ecosystem of teachers and games companies. Capabilities include multi-modal sensors to observe emotional affect, game interaction and decisionmaking. Information is acquired through standard protocols (e.g. XAPI [2]) and evaluated by learning analytics algorithms to provide real-time feedback on player behaviours that are be used for in-game feedback and adaptation, and by teachers to shape follow-up activities. The platform infrastructure is deployed at European schools to gather evidence for effectiveness, to promote to policy makers and to increase adoption of game-based learning in schools. The platform considers how to support interactions between different users of the platform (teachers and games companies). Games companies produce value by offering a range of social games to teachers for evaluation and incorporation into lessons.

Games are developed using the platform API that aims at accelerating game development by supporting a learning process through generic capabilities for student observation and fusion, game interaction monitoring, social learning analytics, feedback and adaptation and access to the educational game marketplace.

Through these capabilities, games using the platform support a learning analytics pipeline that transforms student monitoring and observations into actionable insights for teachers as part of reflection and feedback activities, or for dynamic intelligent adaptation of the game itself. Three analytics channels are combined to provide knowledge of a player's prosocial behaviour (Fig. 2). At runtime skill measurements are acquired through game interaction monitoring whilst temporal emotional state is observed through multi-modal sensors analysing voice and facial expression. The data is stored across multiple games as part of a learning record and combined with off-line questionnaires capturing additional information such as demographics and cultural context.

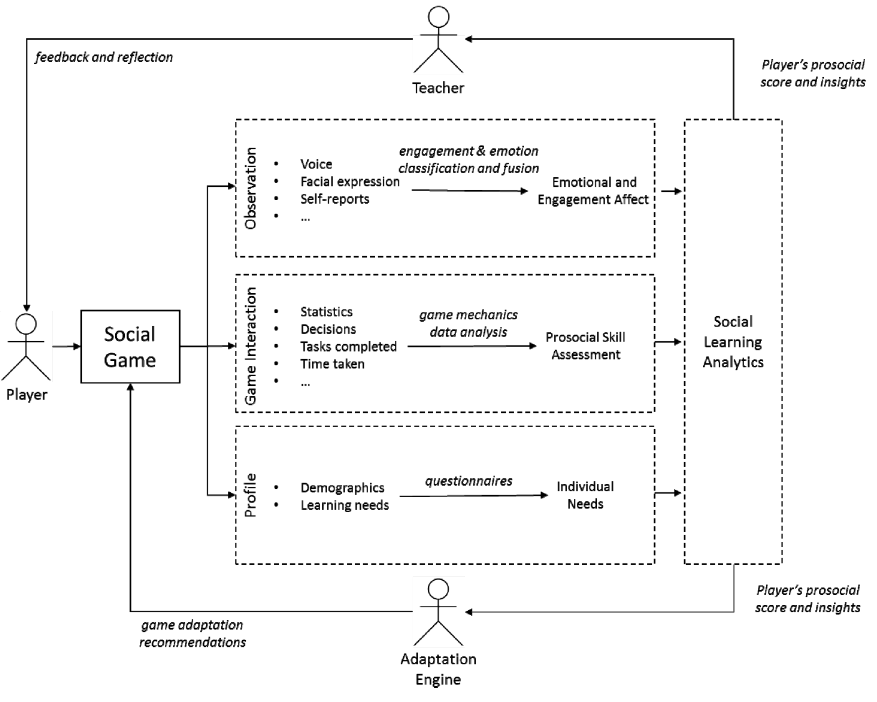

Fig. 2 Prosocial learning analytics pipeline

\section{PROSOCIALLEARN INFORMATION SYSTEMS ARCHITECTURE}

The architecture is composed of loosely coupled services each providing a coherent set of functionality contributing towards management and delivery of prosocial learning experiences. Each subsystem offers APIs that contribute to the full Prosocial API with respective data management and user interface components. A description of each subsystem is shown in Table I.

TABLE I. DESCRIPTION OF PROSOCIALLEARN SUBSYSTEMS

\begin{tabular}{|c|c|}
\hline Subsystem & Functional description \\
\hline $\begin{array}{l}\text { Account } \\
\text { Management }\end{array}$ & $\begin{array}{l}\text { Provides functions to establish business relationships } \\
\text { between platform users and the platform provider }\end{array}$ \\
\hline $\begin{array}{l}\text { User } \\
\text { Management }\end{array}$ & $\begin{array}{l}\text { Provides functions to manage platform user roles and } \\
\text { associated groups }\end{array}$ \\
\hline $\begin{array}{l}\text { Learning } \\
\text { Resource } \\
\text { Management }\end{array}$ & $\begin{array}{l}\text { Provides functions to manage the lifecycle of learning } \\
\text { resource artefacts including deployment, testing, and } \\
\text { certification. }\end{array}$ \\
\hline Marketplace & $\begin{array}{l}\text { Provides functions to publish, search and buy learning } \\
\text { units }\end{array}$ \\
\hline $\begin{array}{l}\text { Lesson } \\
\text { management }\end{array}$ & $\begin{array}{l}\text { Provides functions to manage lessons based on learning } \\
\text { units }\end{array}$ \\
\hline $\begin{array}{l}\text { Learning } \\
\text { Analytics }\end{array}$ & $\begin{array}{l}\text { Provides functions to assess students learning performance } \\
\text { during lessons and retrospectively }\end{array}$ \\
\hline $\begin{array}{l}\text { Player Affect } \\
\text { Observation }\end{array}$ & $\begin{array}{l}\text { Provides functions to observe and measure prosocial } \\
\text { indicators such as emotion and engagement }\end{array}$ \\
\hline Game & $\begin{array}{l}\text { Provides functions to play digital games that are designed } \\
\text { to teach prosocial skills }\end{array}$ \\
\hline $\begin{array}{l}\text { Security } \\
\text { Management }\end{array}$ & $\begin{array}{l}\text { Provides functions to authenticate and authorise users and } \\
\text { their agents (e.g. services, client applications) }\end{array}$ \\
\hline Support Services & $\begin{array}{l}\text { Provides functions for core distributed system capabilities } \\
\text { such as load balancing, service registry, time } \\
\text { synchronisation and content management }\end{array}$ \\
\hline $\begin{array}{l}\text { Infrastructure } \\
\text { and Deployment } \\
\text { Management }\end{array}$ & $\begin{array}{l}\text { Provides functions to manage infrastructure required to } \\
\text { host platform and game services }\end{array}$ \\
\hline Prosocial API & $\begin{array}{l}\text { The Prosocial API provides a document developer guide } \\
\text { for programming the ProsocialLearn platform building on } \\
\text { the APIs of the platform subsystems }\end{array}$ \\
\hline
\end{tabular}

The platform offers a suite of easy-to-integrate APIs for students' behaviour monitoring, game players' management and game matches adaptation. Prosocial Games, to be managed 
by the ProsocialLearn platform have to follow a well-defined architectural style. Below, a short list of the main characteristics a ProsocialLearn game should have:

ProsocialLearn strategy allows game developers freedom to use their favourite technology (such as Unity 3D or Unreal Engine).

Games are managed by the platform. Therefore, the platform governs (create, start, stop, pause, and destroy) game matches which are instances of games between one or more players. The interaction between the platform and the game is supported through a Restful API.

On the other hand, lessons build up the "connection" between games and players. A Teacher describes the Lesson requirements through a Teacher's dashboard by selecting a Learning Unit and tailoring a Lesson Plan Template (provided by the Game Provider) for a Learning Group. This process includes tailoring group and individual level pro-social learning objectives, selecting an allocation plan for distributing students in the group to game instances, and setting any game specific configuration if needed. The lesson is given a start time and duration which is initially taken from the Lesson Plan metadata.

\section{A. Games Interfaces}

Games, to be properly managed by the platform, must expose a series of interfaces:

/actions/getLessonPlanTemplate: to allow the platform to store the Lesson Plan Template of the game

/actions/setGameState: to set a specific game state;

/actions/getGameState: to get the game state;

/actions/healthcheck: to check if the service is up and running.

/actions/connectPlayer: Interface invoked by the game frontend to set specific info of the match.

\section{B. Game Matches Status}

Games matches are managed in according to a well-defined lifecycle defining the multiple game states.

ProsocialLearn games are multiplayer. The interaction among players during the game play should be transparent to the platform. However, the platform should be notified about any relevant actions taken by the game necessary for measuring player performance and progress towards objectives. Matches pass through well-defined states:

initialization: the game server creates the game match as soon as the first players joins the match. At this point, the match is not started yet. Automatically the match passes to the next state "waitingForPlayers".

waitingForPlayers: players are able to join the game match. The match is not started yet. As soon as all players belonging to the match join the game, the match state passes to "ReadyToStart".

readyToStart: the game is ready to start. Two modalities may be implemented: a) the match automatically passes from
readyToStart to running; b) The Teacher clicks on Start Game and the match passes to the next state "running".

running: the match is running. The match can change state to stopped or paused if the teacher asks for Stopping or Pausing of the match. The match can change state to error if an internal error rises.

stopped when the round is ended or the teacher stops the game for any reason.

paused: the teacher can decide, for any reason, to pause the match.

error: when an error raise during the match play.

\section{Interaction with sensors}

The Player Affect Observation (PAO) subsystem automatically acquires, classifies and fuses player emotion and emotion in relation to game play events. A series of multimodal observation channels are established from input sensors connected to player devices including microphones, cameras and keyboard, in addition to more advanced gaming sensors such as Microsoft Kinect and Leap Motion. Using sensing and classification techniques emotion from voice, facial expression and body language is acquired and then fusion processes applied to provide the emotional state of the user [5][6].

The individual classifiers are supervised and pre-trained on an input set of labelled features. The classifiers' outputs are then fused to make a final decision based upon the input features. In general, fusion can happen at two specific levels: feature level fusion (early fusion) and decision level fusion (late fusion). Early and late fusion approaches mainly differ in the way they combine the results from feature extraction on the various modalities. The latter fuses mono-modal decisions into a multimodal semantic representation rather than a multimodal feature representation. As a result, the fusion of decisions becomes easier, while reflecting the individual strength of modalities. Moreover, the late fusion approaches are able to draw a conclusion even when some modalities are not present in the fusion process, which is hard to achieve in the early fusion approach. In addition, late fusion schemes offer flexibility, in a way that different computational models could be used to different modalities. Recent studies have shown that deep learning networks can be applied at feature level as well as at decision level, being trained directly on raw data or decisions accordingly. In our case, we employ a late fusion scheme, where each intermediate classifier is trained to provide a local decision. In terms of affect, local classifiers return a confidence as a probability in the range of $[0,1]$ in a set of predefined classes. The local decisions are then combined into a single semantic representation, which is further analysed to provide the final decision i.e., the affective state of the user [6]. Player affect observations are modelled in accordance with the OGC Observation and Measurement model according to the JSON implementation profile [3]. Valance-arousal dimensional space is used for all modes of measurement unit for classification of emotional state [4]. 


\section{Game adaptation}

The aforementioned elements are measured through an observations acquisition framework that aims to transform raw signal data into player affect and engagement information. Since engagement is a multifaceted phenomenon with different dimensions, i.e., behavioural, cognitive and affective, we model student engagement using real-time data from both the user and the game. More specifically, we use data from sensors to identify the affective state of players, while we extract features related to their cognitive and behavioural engagement based on the analysis of their interaction with the game. The information on user engagement for a particular game is used to identify the game's elements that rank highest for a particular student's persistent engagement profile for that game. A separate prosociality profile is also maintained for each player to rank players according to their achievements with respect to a number of Prosocial Learning Objectives (PLOs), indicating player's ability to properly use and display specific prosocial behaviours or skills.

A dedicated Prosocial Adaptation Manager (PAM) component is therefore employed to provide personalization capabilities to the game and enhance the prosocial behaviour of the players. The PAM is divided into two parts, namely the offline and online adaptation mechanisms. The distinction concerns whether the processing takes place during gameplay or before, in the loading phase of the game. These two mechanisms aim to personalize the prosocial games towards maximizing the players' engagement during gameplay, or select appropriate settings (i.e., scenarios) for demonstrating prosocial skills acquisition.

Offline adaptation occurs prior to the game launch and aims to match the player's prosocial ability to a game scenario, i.e., pre-launch game conditions (such as difficulty setting, contextual data and story-driven interactions) that appropriately match the player's prosociality ranking. Game scenarios ranking is constantly updated as more and more players attempt, win or fail in achieving a particular scenario's prosocial objectives; offline adaptation therefore matches players to a game setting that is determined (by the total number of players) to be the most appropriate to their current prosociality ranking.

Online adaptation on the other hand is responsible for making dynamic adjustments to the game in order to guide players towards game content they'll most likely enjoy, thus maintaining their engagement high. The adaptive features in this mode of adaptation, e.g., the game elements are best represented in the case of prosocial games as the game's means for administering positive reinforcement and corrective feedback to commend or correct player in-game actions with respect to the prosocial skill they need to demonstrate. Such elements may include among others the use of in-game GUI messages, graphics, audio messages or tutoring avatars.

\section{Prosociallearn MARKET Place}

The ProsocialLearn marketplace provides functions to publish, search and acquire learning units incorporating prosocial games (https://prod-psl.atosresearch.eu/store/). The marketplace is managed by the platform business manager who is responsible for defining marketplace economics through definition of pricing models, revenue models, game provider publication agreements, and learning provider access agreements.

\section{CONCLUSIONS}

This paper discussed the architecture of the ProsocialLearn platform: a digital distribution platform for pro-social digital games. The paper introduces the ProsocialLearn methodology to design and implement digital games aimed at increasing social inclusion and academic performance as well as software facilities and a community to support the creation and delivery of digital games for children (7-10 years old) within educational systems that support learning of prosocial skills. The platform provides an application programming interface (API) framework, which gaming developers can use to integrate many of ProsocialLearn functions into their games, including engagement monitoring, in-game achievements, micro-transactions, etc.

In the context of the project, five game providers (European SMEs) have already developed pro-social games and published them in the ProsocialLearn marketplace. The marketplace has been adopted by several schools in Greece, Italy, Spain and UK.

\section{ACKNOWLEDGMENT}

The work reported in this paper has been funded by the ProsocialLearn project under the grant H2020-ICT-2014$1 / 644204$.

\section{REFERENCES}

[1] McGinnis, E., \& Goldstein, A. P. (1997). Skillstreaming the elementary school child: New strategies and perspectives for teaching prosocial skills: Research Press.

[2] xAPI Specification: https://github.com/adlnet/xAPISpec/blob/master/xAPI.md

[3] Observations and Measurements standard: http://www.opengeospatial.org/standards/om

[4] Russell, J.A. (1980). A Circumplex Model of Affect. Journal of Personality and Social Psychology 39 (6): 1161-78

[5] K. Kaza , A. Psaltis , K. Stefanidis , K. Apostolakis , S. Thermos , K. Dimitropoulos, P. Daras, "Body motion analysis for emotion recognition in serious games", HCI International, Toronto, Canada, July 2016.

[6] A. Psaltis, K. Kaza, K. Stefanidis, S. Thermos, K. Apostolakis, K. Dimitropoulos, P. Daras, "Multimodal affective state recognition in serious games applications", IEEE International Conference on Imaging Systems and Techniques, October 4-6, 2016.

[7] K. C. Apostolakis, K. Stefanidis, A. Psaltis, K. Dimitropoulos, and P. Daras, "Offline and Online Adaptation in Prosocial Games", 9th International Conference on Virtual Worlds and Games for Serious Applications, (VS-Games 2017), Athens, Greece, 6-8 September 2017.

[8] ProsocialLearn deliverable D1.1 - Market And Competition Analysis http://prosociallearn.eu/wp-content/uploads/2015/10/D1.1Market\%20And\%20Competition\%20Analysis-Final\%20version.pdf 